\title{
LEVANTAMENTO E IDENTIFICAÇÃO DOS SÍMBOLOS GRÁFICOS UTILIZADOS PARA A CARACTERIZAÇÃO DE CONTROLES E COMANDOS EM MÁQUINAS AGRÍCOLAS
}

\author{
AIRTON DOS S. ALONÇO ${ }^{1}$, MÔNICA R. G. BALESTRA ${ }^{2}$, VILNEI DE O. DIAS ${ }^{3}$, \\ FABRÍCIO A. MEDEIROS ${ }^{4}$
}

\begin{abstract}
RESUMO: Os símbolos gráficos utilizados para indicar a função dos comandos e controles das máquinas agrícolas tornam a operação mais segura. Logo, este trabalho teve por objetivo identificar a simbologia existente nas máquinas agrícolas disponíveis nas quatro principais concessionárias de Santa Maria - RS. No levantamento, foram utilizados como parâmetros os símbolos gráficos recomendados pelas normas técnicas, sendo os existentes nas máquinas estudadas fotografados e posteriormente anotados em planilha. O resultado encontrado foi, em média, de $86 \%$, em relação à utilização dos símbolos gráficos recomendados pelas normas, porém existe também a utilização de símbolos gráficos criados pela própria empresa, o que pode gerar dúvidas no operador da máquina agrícola, pois nem sempre o significado dos mesmos coincide com o descrito nas normas. Os resultados demonstraram que os fabricantes das máquinas agrícolas utilizam as normas existentes, mas ainda não o fazem de forma correta e homogênea.
\end{abstract}

PALAVRAS-CHAVE: ergonomia, normas, símbolos gráficos.

\section{CLASSIFICATION AND IDENTIFICATION OF THE GRAPHIC SYMBOLS USED FOR THE CHARACTERIZATION OF CONTROLS AND COMMANDS IN AGRICULTURAL MACHINES}

\begin{abstract}
The graphic symbols that indicate the function of the commands and controls of the agricultural machines, keep its safer operation. Therefore, this work had as objective, the identification of the existent ones in the available agricultural machines in the four principal concessionary of Santa Maria City - RS, Brazil, the rising were used as parameter, the graphic symbols recommended in the standards, and the existent ones in the studied machines were photographed and later logged in a spreadsheet. The conformities and nonconformities found were contained in a table for better visualization. The result found was average of $86 \%$, in relation to the use of the graphic symbols recommended by the standards, however, there is the use of graphic symbols also created by the own company, what generates doubts in the operator of the agricultural machine, because not always the meaning of the same ones coincides with described in the standards. The results demonstrated that the fabricator of agricultural machines studied use the existent standards, fact this that leads the equality lack among the market placed products.
\end{abstract}

KEYWORDS: ergonomics, standards, graphic symbols.

\footnotetext{
${ }^{1}$ Eng $^{\text {o }}$ Agrícola, Prof. Adjunto, Departamento de Engenharia Rural, CCR, UFSM, Santa Maria - RS, alonço@ ccr.ufsm.br.

${ }^{2}$ Eng $^{\mathrm{a}}$ Florestal, UFSM, Santa Maria - RS.

${ }^{3}$ Acadêmico do Curso de Agronomia, UFSM, Santa Maria - RS.

${ }^{4}$ Engo Agrícola, Mestrando do Programa de Pós-Graduação em Engenharia Agrícola, UFSM, Santa Maria - RS.

Recebido pelo Conselho Editorial em: 23-8-2005

Aprovado pelo Conselho Editorial em: 4-5-2006
} 


\section{INTRODUÇÃO}

O crescimento econômico da agricultura brasileira fez com que a evolução tecnológica chegasse ao campo, elevando a produtividade e capitalizando o agricultor. Dessa forma, e com linhas de financiamento adequadas, foi possível iniciar a substituição de máquinas e implementos agrícolas já obsoletos por outros equipamentos mais sofisticados.

De acordo com BUTIERRES (1996), embora os projetistas se preocupem em realizar os seus projetos contendo o máximo de segurança operacional possível, um implemento ou máquina agrícola sempre possuirá pontos de riscos de acidentes. Com isso, o que resta a fazer, em muitos casos, é alertar os operadores para tais perigos, utilizando símbolos, sinais sonoros, luminosos, com cores características, e palavras de alerta, entre outros, gerando, assim, regras fundamentais de combate aos acidentes de trabalho, que é a sua prevenção.

Muitos são os fatores que contribuem para a ocorrência de acidentes no meio rural. Nesse sentido, um dos fatores que merece destaque, segundo ALONÇO (2004), é a falta de exigência por parte dos usuários na inserção de requisitos de segurança nas máquinas agrícolas utilizadas no Brasil, pois nem sempre quem as adquire é quem as opera. De acordo com MIALHE (1996), os critérios de ergonomia e segurança foram incorporados mais recentemente na formulação de metodologias de ensaio de máquinas agrícolas e há ainda a falta de informação aos projetistas sobre legislação, normas regulamentadoras e normas técnicas.

Com o advento da modernização do meio rural, surge a preocupação de criar meios para que se obtenham qualidade e homogeneidade nas máquinas e equipamentos oferecidos. Assim, foram criadas normas técnicas para projetos de máquinas e implementos agrícolas que, como especifica BACK (1983), "Norma" é uma referência, resultante de uma escolha coletiva arrazoada, visando a servir de base a entendimentos repetitivos.

Baseado nas informações contidas nos parágrafos anteriores, foi constatado que existem normas brasileiras e internacionais que determinam a utilização de símbolos gráficos para a identificação de comandos e controles de máquinas e implementos agrícolas. Frente à crescente modernização do maquinário no meio rural, a Associação Brasileira de Normas Técnicas (ABNT) reuniu, na NBR 11379 (ABNT, 1990), 95 símbolos gráficos para máquinas agrícolas, sendo alguns desses existentes também na ISO 3767/1 (1982), ISO 3767/2-1 (1982), ISO 11684 (1995) e ASAE 5304.5 (1984), de modo a permitir que a indústria brasileira também utilize os mesmos de forma normalizada, oportunizando, ainda, que os equipamentos sejam exportados sem causar constrangimento aos fabricantes em não atender ao que determina a legislação dos países importadores.

A aplicação das normas técnicas em projetos de máquinas agrícolas, inicialmente, cria uniformidade entre os fabricantes, pois o significado dos símbolos é o mesmo para todas as máquinas agrícolas, proporcionando ao operador segurança para realizar o procedimento correto de utilização dos controles e comandos e garantindo ao fabricante a certificação de que seu produto atende às mesmas.

Este trabalho teve por objetivo identificar nas máquinas agrícolas, hoje comercializadas no Brasil, a presença dos símbolos gráficos recomendados pela NBR 11379 (ABNT, 1990) e pela ISO 11684 (1995), e em qual proporção são utilizados e, com base nessas informações, realizar uma comparação entre os símbolos recomendados e os símbolos utilizados pelas empresas e quais seus significados.

Esta pesquisa ganha em importância na medida em que visa também a buscar junto ao instrumento de trabalho dos operadores de máquinas agrícolas, quais as reais informações de que dispõem, partindo-se do princípio de que profissionais bem informados farão operações mais seguras, minimizando, assim, riscos de acidentes de trabalho com máquinas e implementos agrícolas. Foi também objetivo deste trabalho, oferecer subsídios aos fabricantes para a 
uniformização dos símbolos por meio da divulgação da existência das referidas normas técnicas, possibilitando, dessa forma, reduzir os acidentes de trabalho com máquinas agrícolas no meio rural.

\section{MATERIAL E MÉTODOS}

O trabalho foi desenvolvido junto às quatro principais concessionárias de máquinas agrícolas de Santa Maria - RS (Itaimbé Máquinas, Super Tratores Máquinas Agrícolas Ltda., Verdes Vales Máquinas e Equipamentos Agrícolas Ltda. e Valtra Ltda.), de agosto a novembro de 2004, sendo analisadas as máquinas agrícolas disponíveis para venda nesse período de estudo. Depois de identificados os símbolos existentes nas máquinas agrícolas, por meio de avaliação visual, registro em fotografias e consulta a seus manuais, foi realizada a comparação dos símbolos encontrados com os existentes na NBR 11379 (1990) e na ISO 11684 (1995), permitindo analisar em que proporção os mesmos foram encontrados. Os materiais utilizados neste trabalho foram: uma máquina fotográfica, manuais das máquinas agrícolas, normas técnicas NBR 11379 (1990) e ISO 11684 (1995) e uma planilha desenvolvida para este trabalho. Dessa forma, nessa planilha, foi registrada a simbologia presente em cada máquina agrícola, marcados os símbolos encontrados, que correspondiam aos das normas técnicas citadas, e nas observações, eram anotados os símbolos gráficos criados pelo fabricante, não previstos nas normas.

Das máquinas disponíveis nas concessionárias, o estudo concentrou-se, principalmente, em tratores e colhedoras. Sendo este um trabalho acadêmico, sem objetivo de julgar as empresas fabricantes dos equipamentos ou suas concessionárias, referem-se às mesmas utilizando apenas a denominação de Empresa A, Empresa B, Empresa C e Empresa D.

\section{RESULTADOS E DISCUSSÃO}

Os resultados das conformidades e não-conformidades em relação aos símbolos gráficos recomendados pela NBR 11379 (1990) e pela ISO 11684 (1995) são demonstrados na Tabela 1.

Das 25 máquinas agrícolas analisadas, o modelo $\mathrm{n}^{\mathrm{0}}$ 6, da Empresa A, foi o que apresentou menor número de conformidades, enquanto o modelo 21, da Empresa C, apresentou o maior índice de conformidades, sendo a média geral de conformidades de $86 \%$ do total de símbolos gráficos encontrados nas mesmas.

Na Figura 1, são apresentados desde símbolos que não constam no manual do operador, bem como palavra em inglês que, segundo a NBR 5530 (1992) que trata sobre estrangeirismos, "em princípio, devem-se evitar palavras desde que exista palavra correspondente em português, embora de uso não-generalizado. O uso, entretanto, pode sobrepujar razões de ordem puramente etimológica". Parece não ser esse o caso encontrado, visto que a palavra "pare" é bastante comum no vocabulário português. Segundo o manual do operador, os símbolos existentes na Figura 1 significam da esquerda para a direita: motor parado e acessórios desconectados, contato fechado e acessórios conectados, pré-aquecedor da partida ativado, e o último símbolo, lamentavelmente, não foi encontrado no manual do operador, impossibilitando que fosse conhecido seu significado e função.

Segundo a NBR 11379 (1990), as cores utilizadas para indicação e advertência devem ser: verde, amarela e vermelha (Figura 2), e não como apresentada na Figura 3, o que permite ao operador interpretar erroneamente a temperatura da água. 
TABELA 1. Resultados encontrados na pesquisa das máquinas agrícolas.

\begin{tabular}{cccccc}
\hline \multirow{2}{*}{ Modelo } & Empresa & $\begin{array}{c}\text { Potência no } \\
\text { motor }(\mathrm{cv})\end{array}$ & $\begin{array}{c}\text { Número Total de } \\
\text { Símbolos Observados }\end{array}$ & $\begin{array}{c}\text { Conformidade } \\
(\%)\end{array}$ & $\begin{array}{c}\text { Não-conformidade } \\
(\%)\end{array}$ \\
\hline 1 & & 75 & 17 & 88,2 & 11,8 \\
2 & 85 & 21 & 85,7 & 14,3 \\
3 & & 106 & 25 & 92 & 8 \\
4 & & 121 & 28 & 92,9 & 7,1 \\
5 & $\mathrm{~A}$ & 140 & 35 & 74,3 & 25,7 \\
6 & & 150 & 29 & 68,9 & 31,1 \\
7 & & 180 & 24 & 91,7 & 8,3 \\
8 & & 180 & 28 & 82,1 & 17,9 \\
9 & & 225 & 24 & 79,2 & 20,8 \\
\hline 10 & & 78 & 24 & 91,7 & 8,3 \\
11 & & 104 & 28 & 89,3 & 10,7 \\
12 & & 104,4 & 28 & 89,3 & 10,7 \\
13 & $\mathrm{~B}$ & 125 & 29 & 93,1 & 6,9 \\
14 & & 137 & 33 & 90,9 & 11,8 \\
15 & & 140 & 34 & 88,2 & 22,9 \\
16 & & 170 & 35 & 77,1 & 18,7 \\
\hline 17 & & 72 & 14 & 81,3 & 11,8 \\
18 & & 75 & 17 & 88,2 & 18,2 \\
19 & & 87 & 20 & 81,8 & 10 \\
20 & $\mathrm{C}$ & 104 & 20 & 90 & 5 \\
21 & & 120 & 20 & 95 & 12,5 \\
22 & & 130 & 15 & 80 & 16,6 \\
23 & & 175 & 24 & 83,00 \\
\hline 24 & & 190 & 29 & 86,00 & \\
\hline Média & & 75 & & & 5 \\
\hline
\end{tabular}

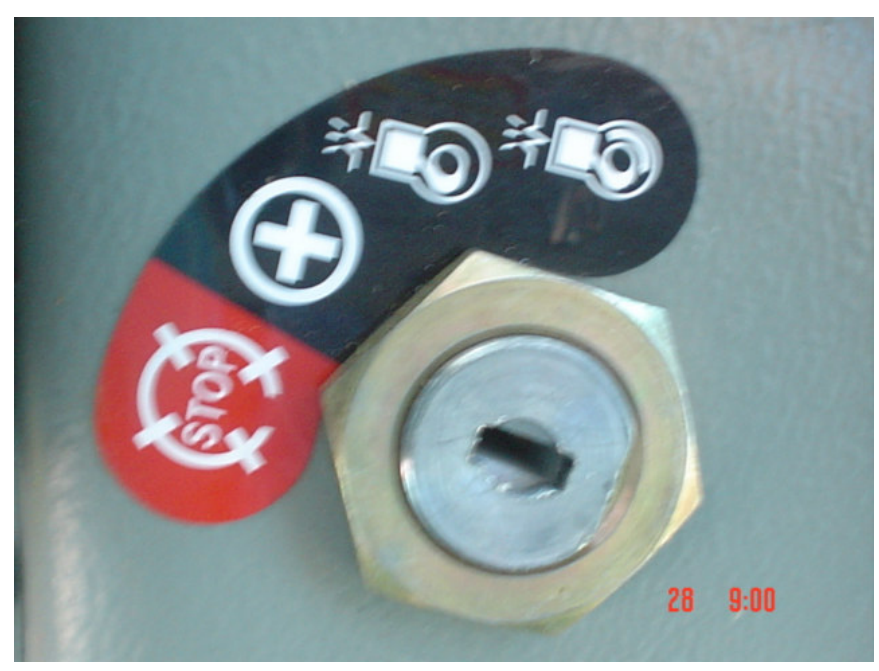

FIGURA 1. Utilização de símbolos inexistentes na NBR 11379 (ABNT, 1990) e ISO 11684 (1995). 


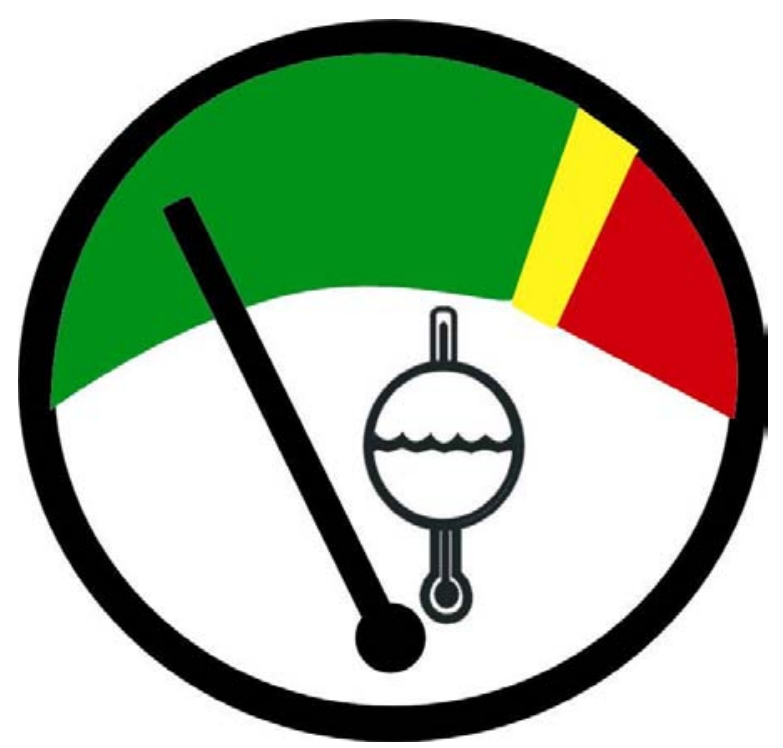

FIGURA 2. Cores recomendadas pela NBR 11379 (ABNT, 1990) para o indicador da temperatura da água que circula pelo sistema de arrefecimento do motor.

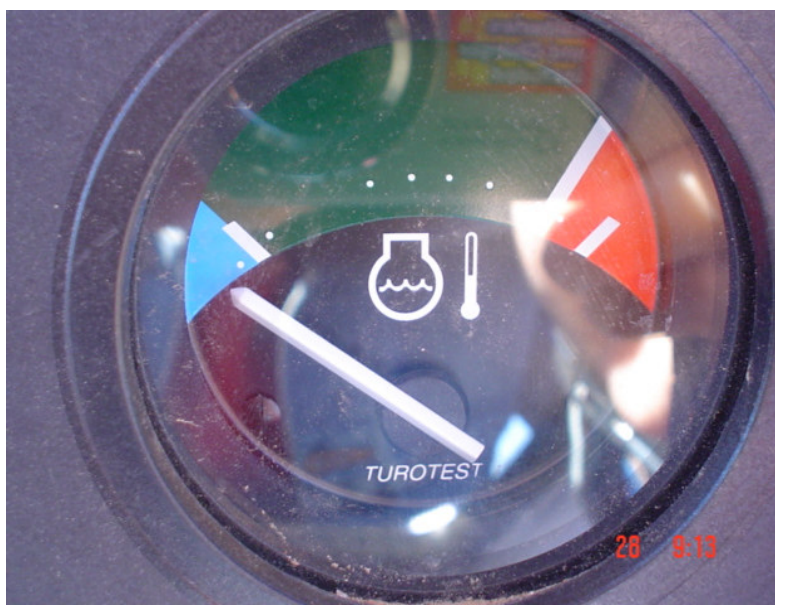

FIGURA 3. Cores utilizadas de forma incorreta para o indicador da temperatura da água que circula pelo sistema de arrefecimento do motor, por um dos equipamentos comercializados pela concessionária $\mathrm{B}$.

Na NBR 11379 (ABNT, 1990), existem dois símbolos gráficos recomendados para indicar tomada de potência engatada e desengatada (Figura 4), porém, como pode ser visto na Figura 5, foi necessária a utilização de seis símbolos gráficos para indicar o mesmo significado de execução, sendo mais fácil e menos oneroso ao fabricante utilizar os símbolos recomendados pela norma, que são somente dois.

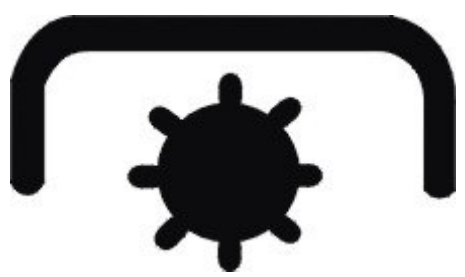

Tomada de potência - desengatar

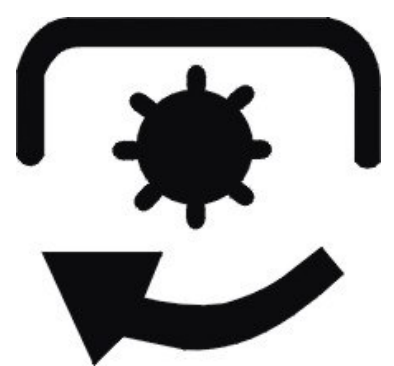

Tomada de potência - engatar

FIGURA 4. Símbolos gráficos recomendados para indicar a tomada de potência engatada e desengatada. Fonte: NBR 11379 (ABNT, 1990) 


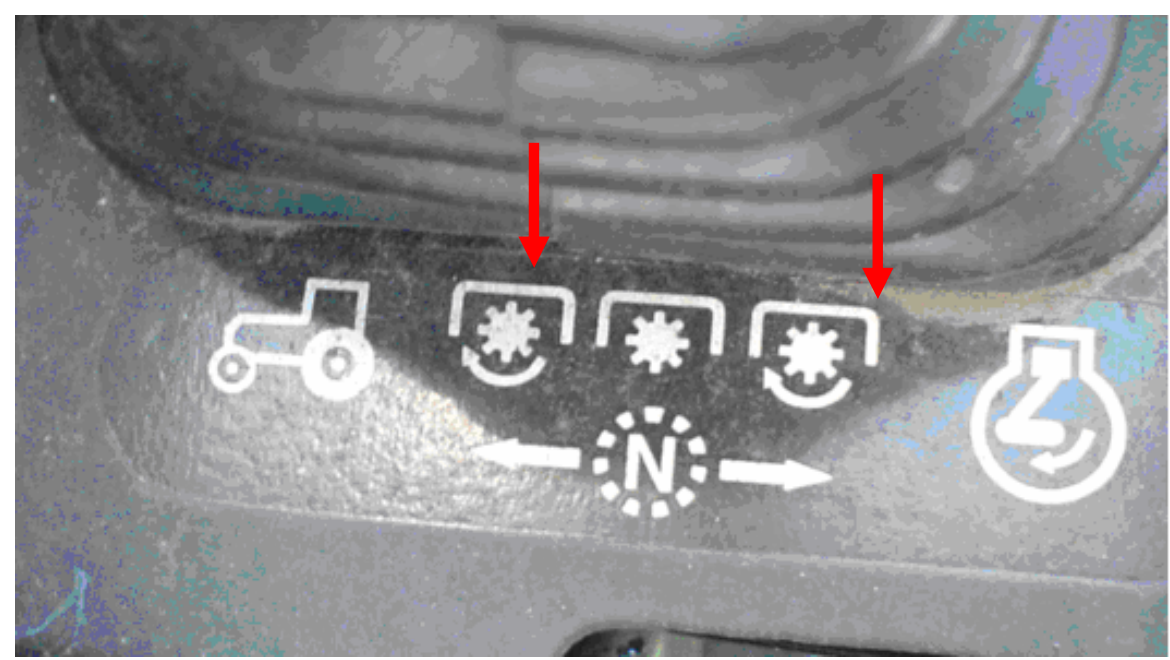

FIGURA 5. Uso excessivo de símbolos para indicar apenas duas funções.

Em muitos casos, também foi constatada a inexistência de símbolos gráficos nos comandos e controles (Figura 6), proporcionando o fato de que o operador tenha de "descobrir" para que serve tal comando, dificultando a operação da máquina agrícola, podendo levar o operador a executar a tarefa errônea ou equivocadamentemente, gerando riscos ao mesmo, ao ambiente e à própria máquina.

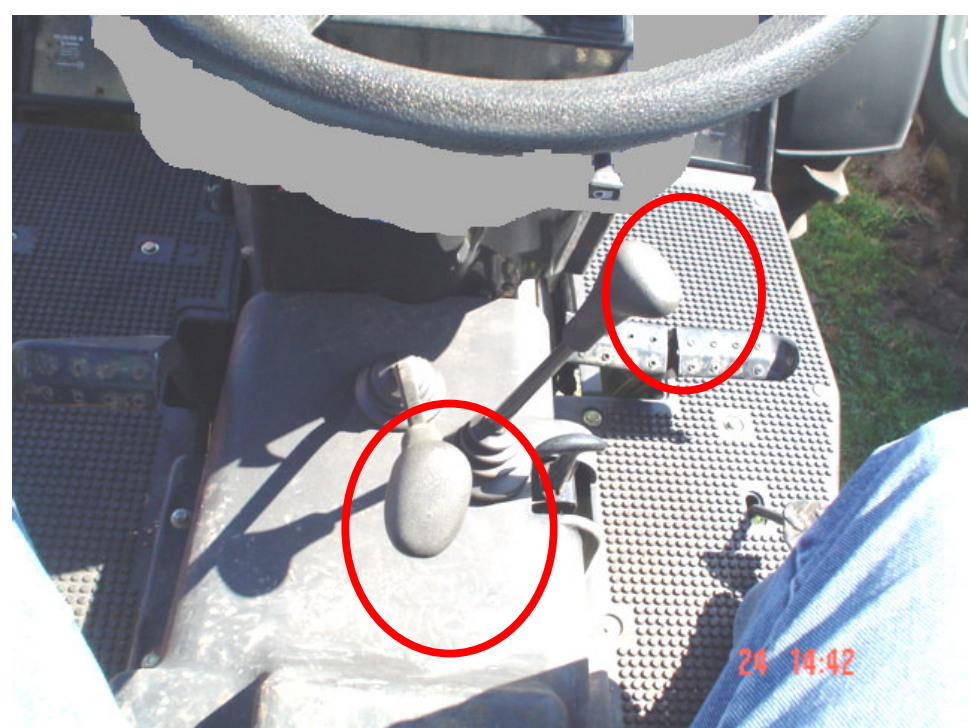

FIGURA 6. Inexistência de símbolos gráficos nos comandos da máquina.

Outro aspecto de não-conformidade importante encontrado neste trabalho foi a constatação do uso de símbolos semelhantes aos símbolos gráficos das normas técnicas, porém com outros significados (Figura 7), o que pode condicionar o operador a executar tarefas erroneamente.

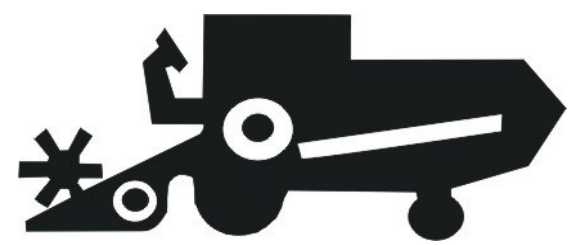

Totalmente mecanizado

NBR 11379 (ABNT, 1990)

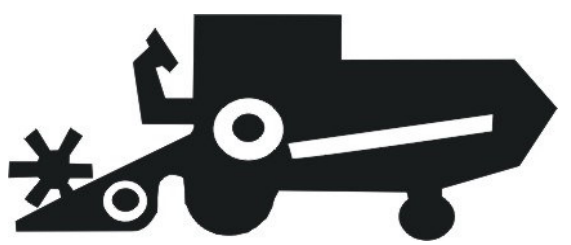

All mechanisms

ASAE S304.5 (1984)

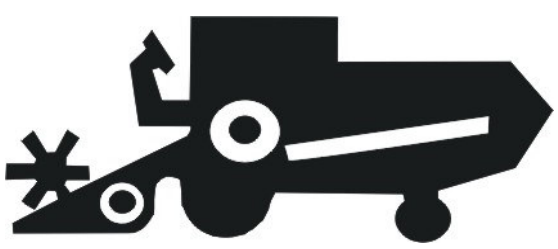

Alavanca de acionamento da transmissão da trilha Manual do operador

FIGURA 7. Símbolos semelhantes com significados diferentes. 
Nesse sentido, outra não-conformidade que merece destaque é a criação de novos símbolos, por parte das empresas, para definir funções que já foram especificadas anteriormente pelas normas técnicas já existentes (Figura 8) e que ajudam ainda mais a gerar dúvidas e atos inseguros por parte dos usuários das máquinas agrícolas.

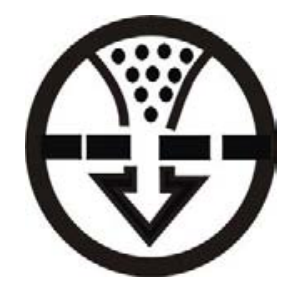

Fonte: NBR 11379

(ABNT, 1990)

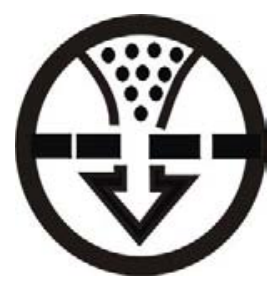

Fonte: ISO 3767/1 (1982)

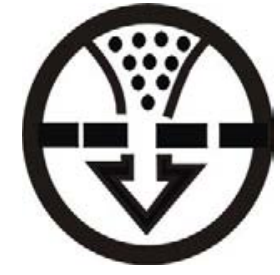

Fonte: ASAE S304.5 (1984)

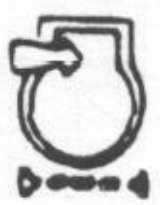

Significado: Filtro de ar seco

Fonte: Empresa B

FIGURA 8. Exemplo da criação de novo símbolo por parte das empresas.

\section{CONCLUSÕES}

Constatou-se que nenhuma máquina agrícola estudada apresentou total conformidade com as normas técnicas, aspecto que proporciona confusão e dúvidas quanto ao significado de cada símbolo. Além disso, em alguns casos, a falta de indicação nas alavancas de alguns modelos demonstraram que nem sempre as empresas estão preocupadas com a execução de operações seguras, fato esse verificado em diversos comandos e controles das máquinas estudadas.

Com isso, o uso de símbolos gráficos das Normas NBR 11379 (ABNT, 1990) e ISO 11684 (1995) não é utilizado com naturalidade pelos fabricantes, talvez por falta de conhecimento por parte dos projetistas. Ainda assim, julga-se que deveria haver maior interesse dos fabricantes em disponibilizar, nos seus equipamentos, símbolos gráficos uniformes, pois, além de atenderem aos interesses dos usuários, que poderão operar e realizar manutenção de máquinas agrícolas de diferentes marcas, o fabricante poderá ter seu produto inserido sem sobressaltos em outros mercados consumidores mais exigentes e esclarecidos.

\section{REFERÊNCIAS}

ALONÇO, A. dos S. Metodologia de projeto para a concepção de máquinas agrícolas seguras. 2004. 221 f. Tese (Doutorado em Projetos de Sistemas Mecânicos) - Universidade Federal de Santa Catarina, Florianópolis, 2004.

AMERICAN SOCIETY OF AGRICULTURAL ENGINEERS. ASAE 5304.5: Symbols for operator controls on agricultural equipment. St. Joseph, 1984. 4 p.

ASSOCIAÇÃO BRASILEIRA DE NORMAS TÉCNICAS. NBR 11379: Símbolos gráficos para máquinas agrícolas. São Paulo, 1990. 13 p.

ASSOCIAÇÃO BRASILEIRA DE NORMAS TÉCNICAS. NBR 5530: Elaboração e aplicação da terminologia de veículos rodoviários e industriais, máquinas rodoviárias, tratores agrícolas e similares. São Paulo, 1992. 3 p.

BACK, N. Metodologia de projeto de produtos industriais. Rio de Janeiro: Guanabara Dois, 1983. $389 \mathrm{p}$.

BUTIERRES, E. Normalização de adesivos de segurança para uso em máquinas agrícolas. In: WORSHOP SOBRE MECANIZAÇÃO AGRÍCOLA NA REGIÃO DE CLIMA TEMPERADO, 2., 1996, Pelotas. Anais... Pelotas: UFPEL/FAEM, EMBRAPA/CPACT, UFSM/CT, 1996. p.1313. 
INTERNATIONAL ORGANIZATION FOR STANDARDIZATION. ISO 3767/1: Tractors machinery for agriculture and forestry, powered lawn and garden equipament - symbols for operator controls and other displays - Part 1: Common symbols. Geneva, 1982. 5 p.

INTERNATIONAL ORGANIZATION FOR STANDARDIZATION. ISO 3767/2-1: Tractors machinery for agriculture and forestry, powered lawn and garden equipament - symbols for operator controls and other displays - Part 2: Symbols for agricultural tractors an machinery. Geneva, 1982. 3 p.

INTERNATIONAL ORGANIZATION FOR STANDARDIZATION. ISO 11684. Tractors, machinery for agriculture and forestry, powered lawn and garden equipment - safety signs and hazard pictorials: general principles. Geneva, 1995. 52 p.

MIALHE, L.G. Máquinas agrícolas: ensaios e certificações. Piracicaba: FEALQ, 1996. 451 p. 